\title{
Comparaison de techniques pratiques de diagnostic de la tuberculose bovine
}

\author{
par J. M. BLANCOU $(*)$
}

\begin{abstract}
RESUME
Différentes méthodes de diagnostic pratique de la tuberculose bovine ont été comparées. La méthode de précipitation interfaciale détecte 40 p. 100 des malades (avec 10 p. 100 d'excès), celle de la précipitation en gélose 37 p. 100 (sans excès), celle de l'hémagglutination indirecte 34 p. 100 (12 p. 100 d'excès), celle de la tuberculose intradermique 96 p. 100 (16 p. $100 \mathrm{~d}$ excès), celle de la tuberculination intravenneuse 48 p. 100 (4 p. 100 d'excès), celle de la tuberculınation sous-cutanée 94 p. 100 (10 p. 100 d'excès). Cette dernière méthode peut être simplifiée (un seul relevé thermique) mais elle ne détecte plus alors que 80 p. 100 des malades.
\end{abstract}

\section{INTRODUCTION}

Le diagnostic de la tuberculose bovine est actuellement réalisé, dans le cadre de la prophylaxie sanitaire de nombreux pays, par la méthode de tuberculination intradermique. Bien que cette méthode donne des résultats excellents, elle présente des défaillances dues en particulier au phénomène d'anergie (4).

En élevage extensif tropical, un second défaut de la méthode réside dans la nécessité de l'appliquer en deux temps (injection-lecture 72 heures plus tard) : elle s'accommode mal des difficultés de rassemblement et contention des bovins et de la réticence naturelle des éleveurs envers l'opération.

A Madagascar enfin, où la tuberculose frappe environ le quart du cheptel bovin (6), les deux problèmes coexistent: difficulté des enquêtes en brousse par la tuberculination intradermique et impossibilité de dépister les sujets anergiques, contagieux, destinés aux établissements d'élevage ou embouche industrielle.

(') I.E.M.V.T., Laboratoire Central de l'Elevage, B.P. n० 862 , Tananarive.
C'est pour rechercher des méthodes de diagnostic complémentaires de la tuberculination intradermique que nous avons entrepris cette étude.

\section{MATERIEL ET METHODES}

Nous avons expérimenté diverses méthodes immunologiques déjà décrites pour la tuberculose, à condition qu'elles soient d'exécution possible loin du laboratoire.

Nous distinguerons deux types de recherches des anticorps tuberculeux :

\section{A) RECHERCHE DES ANTICORPS IN VITRO}

\section{Méthodes de précipitation}

Nous avons employé deux méthodes différentes :

- précipitation interfaciale, en milieu liquide;

- précipitation (par double diffusion) en milieu gélosé.

\section{Antigène}

Il est, dans les deux cas, obtenu à partir des corps microbiens des voiles de bacilles de Koch, 
cultivés en milieu de Sauton et conservés à $-25^{\circ}$. Après congélation et décongélation brutales successives à $37^{\circ}$, ce " cryolysat " laisse exsuder une certaine quantité de liquide: cet exsudat filtré sur membrane "Seitz Eks I " puis ramené au volume de $100 \mathrm{ml}$ pour 1000 grammes de corps bacillaires essorés constitue l'antigène.

Dans le cas de la précipitation interfaciale, l'antigène est utilisé directement, dans le cas de la précipitation en gélose, il est préalablement lyophilisé puis repris au $1 / 10$ de son volume initial afin de le concentrer sans l'altérer.

\section{Sérums}

Le sang est recueilli soit par ponction veineuse sur l'animal vivant, soit par ponction cardiaque sur les fressures saisies à l'abattoir. Cette dernière méthode a l'avantage de permettre d'établir d'emblée une collection de sérums de malades à différents stades.
Les sérums sont conservés à $-25^{\circ}$, ou, dans les méthodes de " sérologie sur papier ", adsorbés sur papier filtre Chardin en carrés de $10 \times 10 \mathrm{~mm}$, ensuite découpés et conservés en atmosphère sèche. Dans le dernier cas, le sang total peut être utilisé, sans séparation préalable du sérum.

\section{Méthodes}

- Précipitation interfaciale (photo $\mathrm{n}^{\circ}$ 1)

Le sérum est déposé, sous le volume de $0,5 \mathrm{ml}$, à l'aide d'une pipette effilée, au fond d'un tube capillaire constitué par l'extrémité boutonnée d'une pipette Pasteur.

L'antigène, sous le volume de $0,5 \mathrm{ml}$, est ensuite amené délicatement à son contact avec une autre pipette.

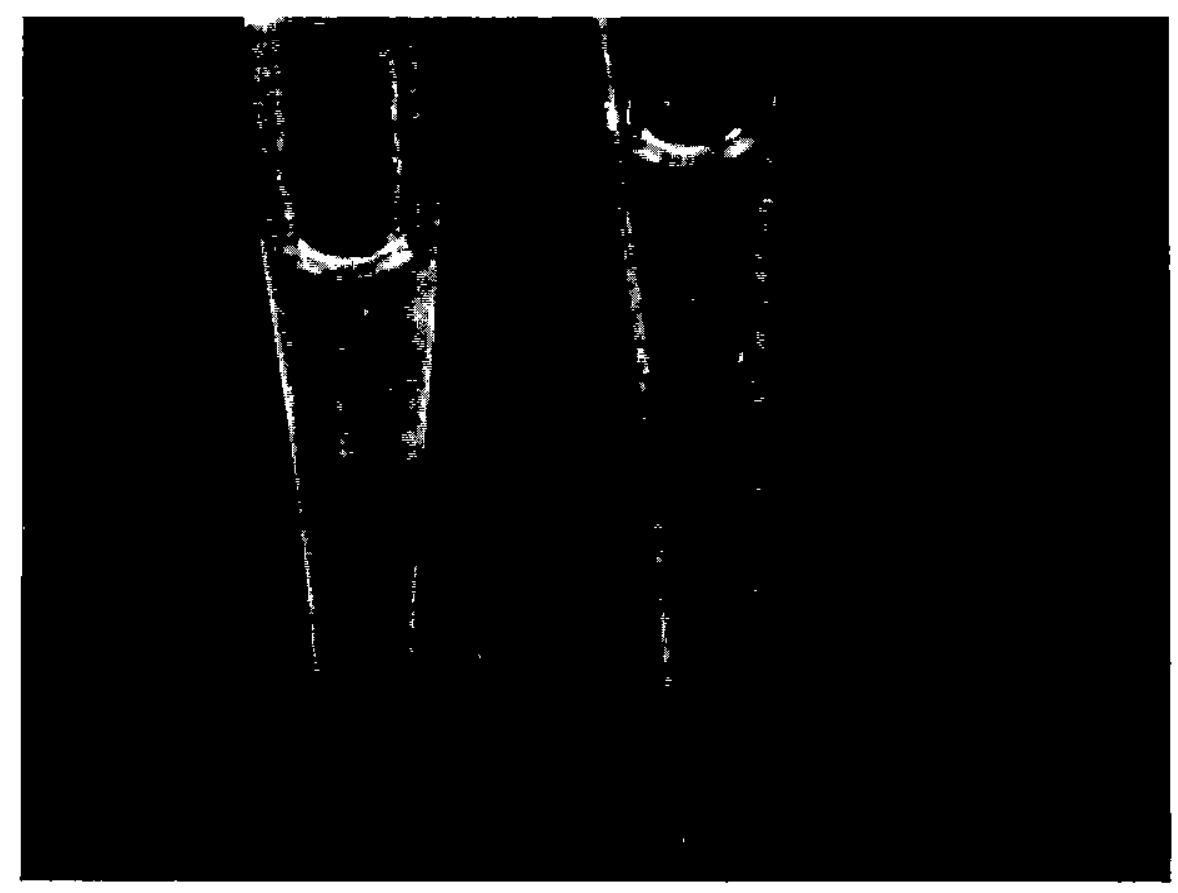

Photo no 1.

Précipitation interfaciale.

- Précipitation en gélose (photos $\mathrm{n}^{\text {os }} 2$ et 3 )

La gélose est coulée sous le volume de $15 \mathrm{mI}$ en boîte de Pétri. Elle a la composition suivante :

$\begin{array}{lll}\text { - Agar noble } & . & 6 \mathrm{~g} \text { (ou } \\ & & \begin{array}{r}\mathrm{g} \text { en sérologie } \\ \text { sur papier). }\end{array} \\ \text { - Merthiolate } & . & 0,1 \mathrm{~g} . \\ \text { - Tampon P.B.S. . } & . & 500 \mathrm{ml} .\end{array}$


L'antigène est déposé soit dans des puits de $6 \mathrm{~mm}$ de diamètre creusés à l'emporte pièce, soit sur du papier Chardin de $10 \times 10 \mathrm{~mm}$, permettant l'étude simultanée de 10 sérums.

\section{Réaction}

La réaction se produit en quelques minutes dans le cas de la précipitation interfaciale et en 12 à 48 heures dans le cas de la précipitation en gélose à la température ambiante.

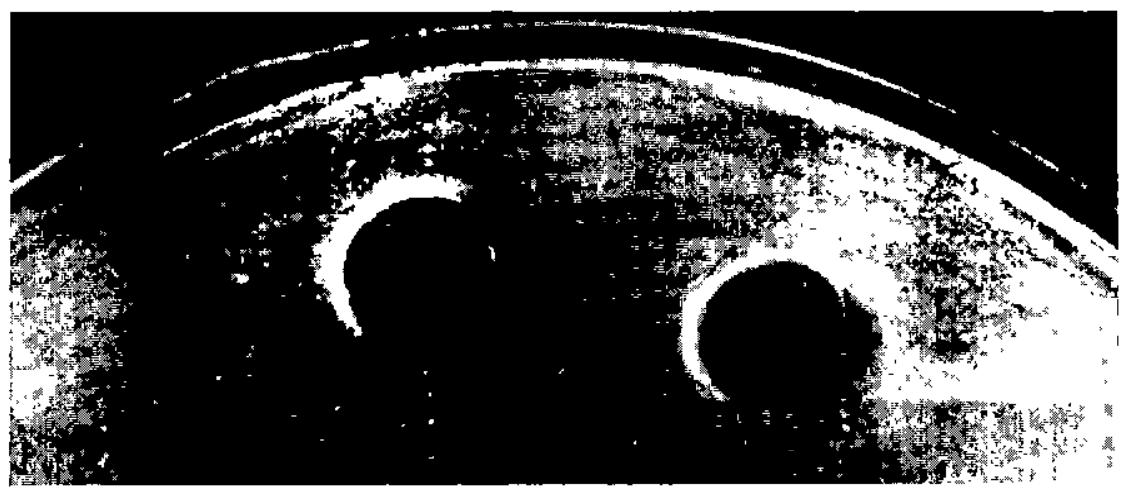

Photo $\mathrm{n}^{\circ} 2$.

Précipitation en gélose (réactifs disposés dans des puits).

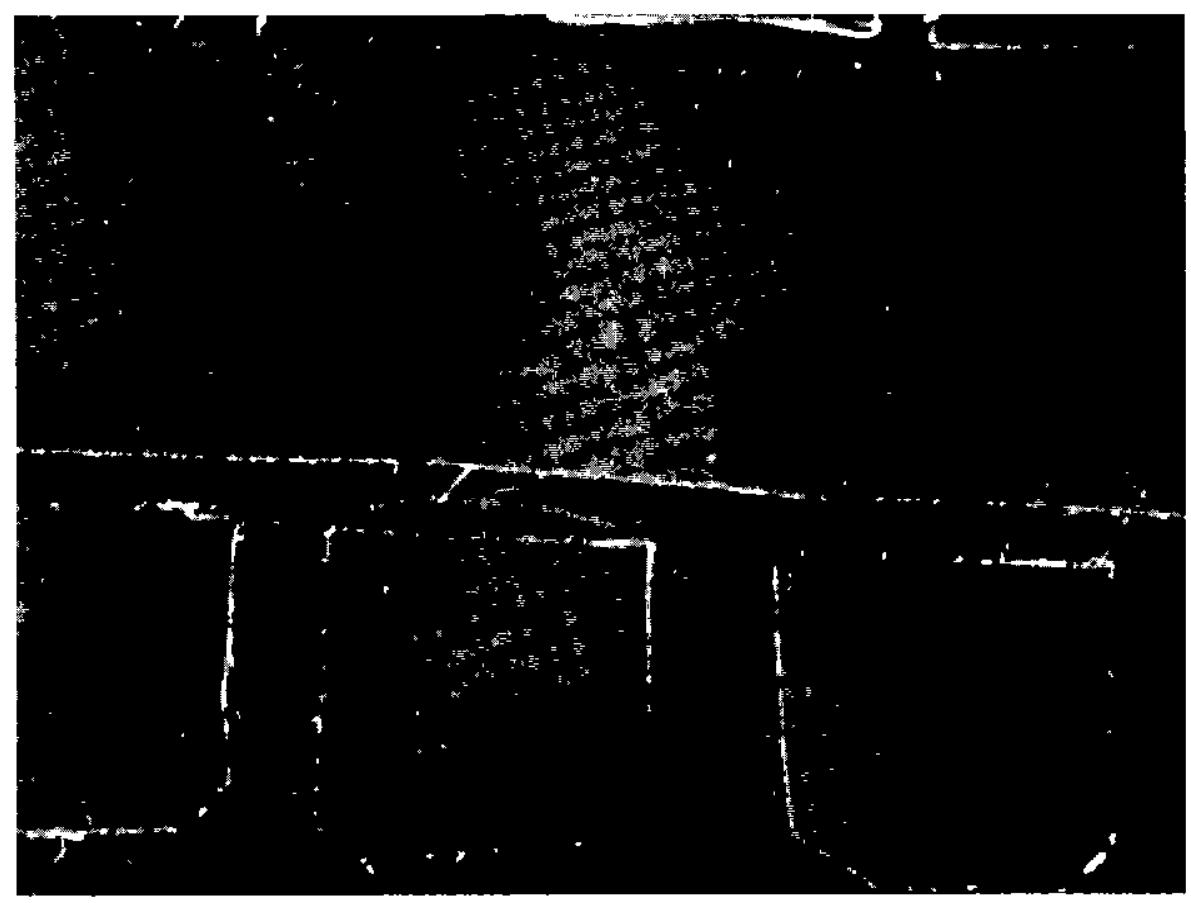

Photo $\mathrm{n}^{\circ} 3$.

Précipitation en gélose (réactifs adsorbés sur papier).

\section{Méthodes d'agglutination} (photo $n^{\circ} 4$ )

Nous n'avons pas utilisé les méthodes d'agglutination directe du bacille (décrite par
ARLOING et COURMONT), G. SIMINTZIS et R. SOHIER (8) ayant démontré « les difficultés insurmontables que l'on éprouve à les transposer dans la pratique courante ». Nous 


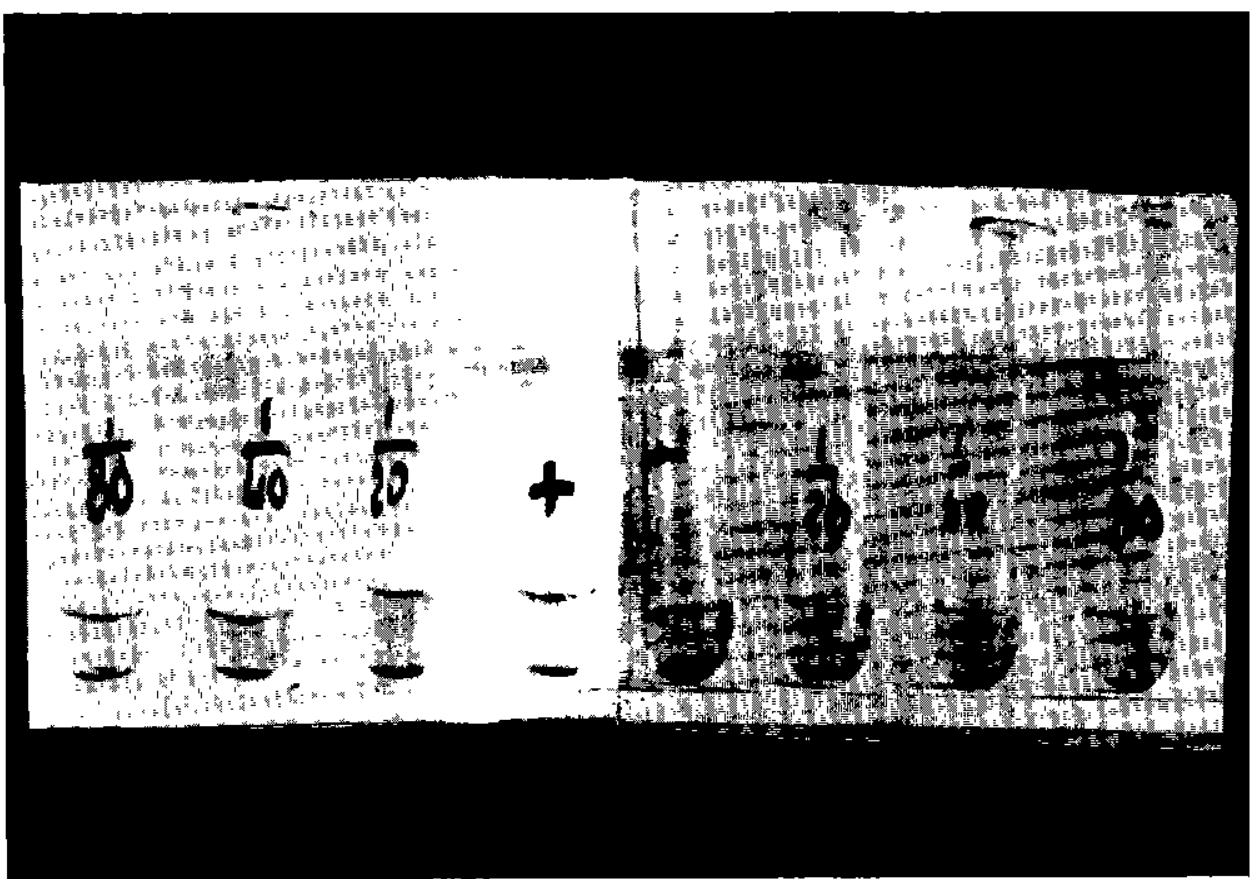

Photo no 4.

Hémagglutination passive (présence d'anticorps dans le sérum + ).

nous sommes attachés par contre à l'étude d'une réaction d'hémagglutination passive du type de celle décrite initialement par MIDDLEBROOK-DUBOS (5) et ensuite largement appliquée au dépistage de la tuberculose bovine par G. SIMINTZIS et de nombreux autres auteurs (7).

L'antigène est identique à celui utilisé dans les méthodes de précipitation. Il peut être utilisé soit directement, soit lyophilisé puis remis en suspension en eau physiologique.

Les hématies sont obtenues par ponction veineuse du mouton, recueillies sur liquide d'Alsewer puis conservées un jour à $+4^{\circ}$ avant leur utilisation.

Les sérums sont recueillis selon les méthodes précédemment indiquées. Ils sont préalablement inactivés à $56^{\circ}$ et absorbés sur hématies de mouton, mais cette pratique peut être évitée car il est très rare que les phénomènes d'agglutination non spécifique disparaissent après ce traitement.

La réaction est exécutée selon le protocole suivant :

1. Dilution des sérums en eau physiologique selon une progression arithmétique, à partir de la solution mère au $1 / 10$, sous le volume de $0,5 \mathrm{ml}$.

2. Sensibilisation des hématies : après avoir été lavées trois fois en eau physiologique, elles sont mises en contact une heure à $37^{\circ}$ (sous le volume de $0,2 \mathrm{ml}$ pour le culot d'hématies) avec $2 \mathrm{ml}$ d'antigène dilué au demi en eau physiologique.

3. Des hématies sensibilisées sont lavées trois fois en eau physiologique puis remises en suspension à 2 p. 100 et distribuées sous le volume de $0,5 \mathrm{ml}$ dans les différentes dilutions du sérum. La réaction se lit après 12 heures à $+4^{\mathrm{p}}$.

Le taux de $1 / 40$ a été choisi comme seuil positif de la réaction.

\section{B) RECHERCHE DES ANTICORPS IN VIVO}

\section{Hypersensibilité retardée : réaction locale}

La recherche de l'hypersensibilité retardée, par injection intradermique de tuberculine à l'encolure, est pratiquée sur environ 30.000 bovins chaque année à Madagascar. Cette tuber- 
culine est préparée selon les méthodes classiques à partir d'une souche de bacille tuberculeux de type bovin (isolé d'un zébu malgache) cultivé en milieu de Sauton. Elle titre 2.500 U.I. $/ \mathrm{ml}$. Les résultats de l'épreuve sont constatés post mortem $(*)$.

\section{Hypersensibilité retardée : réaction générale}

Elle a été recherchée par inoculation de la tuberculine par voie parentérale, intraveineuse ou sous-cutanée.

\section{a) Inoculation intraveineuse de tuberculine}

Cette technique a été longuement étudiée par F. et R. LABORIE, puis d'autres auteurs (2) (3).

La tuberculine, diluée à $1 / 10$, est injectée sous le volume de $10 \mathrm{ml}$ et la température rectale relevée toutes les deux heures. La courbe type d'une telle réaction est représentée sur la figure 1.

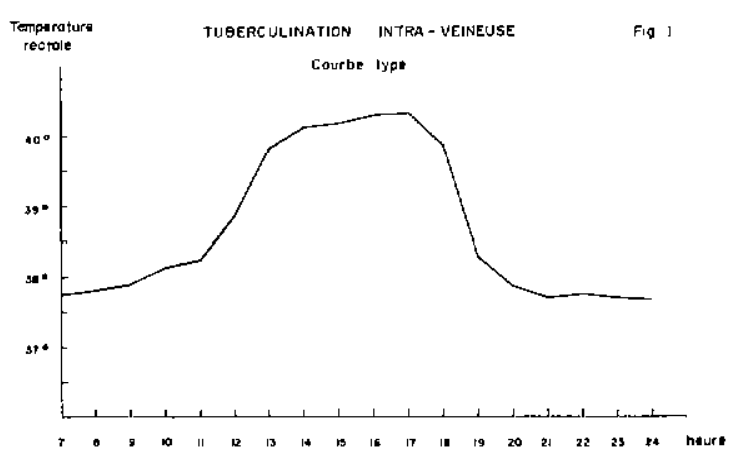

\section{b) Inoculation sous-cutanée de tuberculine}

Nous avons employé la tuberculination souscutanée classique utilisant la dose de $1,5 \mathrm{ml}$ par $100 \mathrm{~kg}$ de poids vif de tuberculine diluée au $1 / 10$. La courbe type d'une telle réaction est représentée sur la figure 2 .

*) Nous remercions vivement notre confrère G. JACQUET du Service Vétérinaire Provincial de Tuléar des renseignements qui nous ont permis de compléter cette étude.

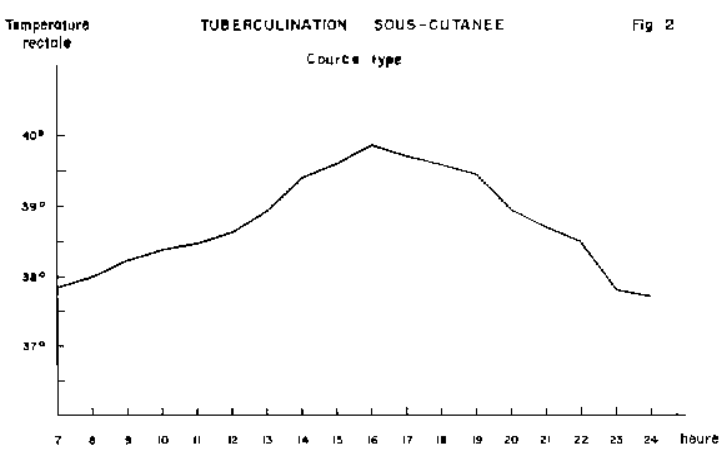

\section{RESULTATS - DISCUSSION}

Nous avons jugé bon de présenter les résultats des diverses réactions pratiquées précédemment en un seul tableau, afin de comparer avantages et inconvénients des diverses méthodes. Le nombre de sujets éprouvés est indiqué entre parenthèses, permettant ainsi d'apprécier les limites de confiance respectives des différentes techniques.

\section{RESULTATS DE LA RECHERCHE DES ANTICORPS IN VITRO}

Les résultats obtenus confirment ceux de la plupart des auteurs (4) et en particulier les importantes expériences de K. ALMASSY (1) : certitude des résultats obtenus par la méthode de précipitation en gélose, incertitude relative de ceux obtenus par les méthodes de précipitation interfaciale et d'hémagglutination passive.

\section{RESULTATS DE LA RECHERCHE DES ANTICORPS $I N$ VIVO}

\section{Tuberculination intradermique}

Cette méthode, très classique, fournit la proportion habituellement constatée (5) de résultats par excès ou par défaut.

\section{Tuberculination intraveineuse}

Nous retrouvons les résultats obtenus par F. et R. LABORIE (2) puis A.B. LARSEN (3). Il semble qu'il existe, dans le cas d'injection de doses importantes de tuberculine par cette voie, une superposition entre les phénomènes d'hypersensibilité immédiate et retardée. Ceci pourrait expliquer le délai parfois très court (quelques heures) d'apparition de la réaction et la discordance entre les tests intradermiques 
(utilisant $1 / 10 \mathrm{ml}$ de tuberculine) et l'injection intraveineuse (utilisant plusieurs millilitres de tuberculine). Cette dernière détecte du reste une forte proportion d' " anergiques " indécelables par l'épreuve intradermique classique.

Son emploi, relativement délicat, peut compléter celui de la tuberculination intradermique mais sans présenter beaucoup plus d'intérêt que la méthode sérologique de précipitation en gélose.

\section{Tuberculination sous-cutanée}

Les résultats positifs indiqués sur le tableau final sont ceux où a été observée une réaction thermique supérieure de $1,5^{\circ}$ à la moyenne des températures relevées avant l'injection. Cette méthode est actuellement délaissée, ou interdite, au profit de la méthode intradermique. Mais en élevage extensif, où la rareté des tuberculinations lève l'hypothèque d'une désensibilisation éventuelle, cette méthode pourrait rendre encore service du fait de la précocité de la réaction.

Nous avons donc recherché s'il n'était pas possible de la simplifier en n'effectuant qu'un seul relevé thermique. Pour cela nous avons recherché, sur quatre-vingt-quatre bovins tuberculeux destinés à l'abattoir, l'heure à laquelle se situait l'écart thermique de $1,5^{\circ}$ considéré comme caractéristique. Cet écart exístait chez 50 p. 100 des sujets dès la $11^{\text {te }}$ heure, chez 75 p. 100 à la $13^{e}$ heure, chez 85 p. 100 à la $16^{\circ}$ heure et chez 45 p. 100 seulement après la $18^{\circ}$ heure.

Nous avons appliqué à plusieurs reprises cette méthode simplifiée dans les conditions pratiques d'élevage extensif, en injectant la tuberculine entre 16 et 17 heures et relevant les températures le lendemain entre 6 et 7 heures. Ces relevés se font par lot de 15 sujets (car l'élévation de la température ambiante modifie les températures rectales) et la température " normale " est déterminée par la moyenne des deux températures les plus basses du lot (un lot n'étant jamais réagissant en totalité). Cette méthode donne des résultats généralement concordants avec ceux de la méthode intradermique, excepté les résultats par défaut signalés $(10-15$ p. 100$)$ et quelques résultats par excès (hyperthermies non spécifiques). Il s'agit donc d'une méthode approchée dont le seul avantage est de n'immobiliser les animaux qu'une seule nuit dans leur parc.

Tableau gënệral des rêsultats

\begin{tabular}{|c|c|c|c|}
\hline \multicolumn{4}{|c|}{$\begin{array}{l}\text { Proportion des rësultats positifs selon les mëthodes } \\
\text { de diagnostic et le type de lésion }\end{array}$} \\
\hline Méthode de diagnostic Types de lésions & $\begin{array}{l}\text { Lésions } \\
\text { d'organes }\end{array}$ & $\begin{array}{l}\text { Lêsions } \\
\text { ganglionnaires } \\
\text { seules }\end{array}$ & $\begin{array}{l}\text { Absence de } \\
\text { lësions } \\
\text { macroscopiques }\end{array}$ \\
\hline $\begin{array}{l}\text { Prêcipitation interfaciale } \\
\text { (sur } 430 \text { sêrums) }\end{array}$ & $25 \mathrm{p} \cdot 100$ & $15 \mathrm{P} \cdot 100$ & $10 \mathrm{p} .100$ \\
\hline $\begin{array}{l}\text { Précipitation en gélose; méthode de puits } \\
\text { (sur } 493 \text { sérums) }\end{array}$ & $35 \mathrm{p} .100$ & $2 \mathrm{p} .100$ & $0 \mathrm{p} .100$ \\
\hline $\begin{array}{l}\text { Précipitation en gélose; méthode sur papier } \\
\text { (sur } 328 \text { sangs complets) }\end{array}$ & $20 \mathrm{p} \cdot 100$ & $1 \mathrm{p} .100$ & 0 p. 100 \\
\hline $\begin{array}{l}\text { Hémagglutination passive } \\
\text { (sur } 395 \text { sérums) }\end{array}$ & $30 \mathrm{p} \cdot 100$ & 4 p. 100 & 12 p. 100 \\
\hline $\begin{array}{l}\text { Tuberculination intra-dermique } \\
(4025 \text { animaux })\end{array}$ & \multicolumn{2}{|c|}{$96 \mathrm{p} .100$} & $16 \mathrm{p} \cdot 100$ \\
\hline $\begin{array}{l}\text { Tuberculination sous-cutanëe } \\
\text { (104 animaux) }\end{array}$ & \multicolumn{2}{|c|}{$94 \mathrm{p} \cdot 100$} & $10 \mathrm{p} \cdot 100$ \\
\hline $\begin{array}{l}\text { Tuberculination intra-veineuse } \\
(25 \text { animaux })\end{array}$ & \multicolumn{2}{|c|}{$48 \mathrm{p} .100$} & 4 p. 100 \\
\hline
\end{tabular}




\section{CONCLUSION}

De la comparaison des résultats obtenus, il ressort que les méthodes n'ont ni la même valeur, ni les mêmes indications.

Pour les enquêtes en élevage extensif: la tuberculine intradermique sera choisie seulement si les éleveurs s'avèrent coopératifs et acceptent de soumettre leurs troupeaux à la visite de contrôle. En région d'accès difficile, la tuberculination sous-cutanée à lecture unique pourra rendre des services, à condition d'admettre quelques résultats par défaut.
Pour le triage des bovins suspects destinés à des élevages intensifs: la tuberculination intradermique sera appliquée d'emblée, puis les bovins suspects d'anergie seront soumis à un examen sérologique (précipitation en gélose). Ces méthodes ne pourront cependant, dépister tous les anergiques contagieux. En tuberculose, comme dans les autres maladies, la méthode idéale de diagnostic est encore à découvrir — et force est de nous contenter, pour l'instant, d'un faisceau de preuves immunologiques parfois utile pour confirmer un diagnostic clinique.

\title{
SUMMARY
}

\section{Comparison between different practical diagnostic tests of tuberculosis in cattle}

\begin{abstract}
Different practical diagnostic tests of tuberculosis in cattle have been compared. Among tubercular cattle, 40 p. 100 gave positive results with the precipitin test (but 10 p. 100 false reaction), 37 p. 100 with the agar gel diffusion test (no false reactions), 34 p. 100 with the passive haemagglutination test (but 12 p. 100 false reaction), 96 p. 100 with the intra-dermal test (16 p. 100 false reaction), 48 p. 100 with the intra-venous test (4 p. 100 false reaction), 94 p. 100 with the sub-cutaneous test $(10$ p. 100 false reaction), but 80 p. 100 if this last test is simplified by taking temperature just once after injection.
\end{abstract}

\section{RESUMEN}

Comparación de técnicas prácticas de diagnóstico de la tuberculosis de los bovinos

Se compararon varios métodos de diagnóstico práctico de la tuberculosis de los bovinos.

El método de precipitación demostra 40 p. 100 de los enfermos (con 10 p. 100 de reacciones falsas), el de precipitación en gelosa 37 p. 100 (sin reacción falsa), el de la hemaglutinación indirecta 34 p. 100 (12 p. 100 de reacciones falsas), el de la tuberculinación intradérmica 96 p. 100 (16 p. 100 de reacciones falsas), el de la tuberculinación intravenosa 48 p. 100 (4 p. 100 de reacciones falsas). el de la tuberculinación subcutánea 94 p. 100 (10 p. 100 de reacciones falsas).

Se puede simplificar el último método, tomando la temperatura una sola vez después de la inyección, pero ya no detecta en tal caso más que 80 p. 100.

\section{BIBLIOGRAPHIE}

1. ALMASSY (K.) - Examination of serum from tuberculin reactors by the gel diffusion test, $M a g$ Allator - Lapja, 1962, 17: 199-202.

- Application of the agar gel-diffusion test to the detection of tuberculotic animals, Prog. immunobiol. Standard, 1964, 1 : 209-212.

2. LABORIE (F.) et LABORIE (R.), A propos de l'actualité de la tuberculination par voie intraveineuse dans l'éradication de la tuberculose bovine, Rec. Méd. vét. Alfort, 1966, 142 (8) : 702-728.

3. LARSEN (A. B.), KOPECKY (K. E.), An intravenous tuberculin test for cattle, Am. J. vet. Res., 1965,26 (112) : 676-678.

4. LUCAS (A.), GAYOT (G.), Pathologie de la production du lait: procédés actuels de dépistage de la tuberculose bovine, Ann. Nutr. Alim., 1967, 21 (1) : 1A-63 A.

5. MIDDLEBROOK (G) et DUBOS (R.), Specific serums agglutination of erythrocytes sensitized with extracts of tubercle bacilli, J. Exp. Med. 1943, 88, 521.

6. Rapports annuels du Service de l'Elevage de Madagascar. Archives de l'I.E.M.V.T., Tananarive.

7. SIMINTZIS (G.), Diagnostic de la tuberculose bovine par la réaction d'hémagglutination: bilan de 10 années d'application, Bull. Soc. Sci. vét. Lyon, 1964, 66 (3) : 233-245.

8. SIMINTZIS (G.), SOHIER (R.), La séro-agglutination de $S$. Arloing et $P$. Courmount dans le diagnostic de la tuberculose bovine, Bull. Acad. vét., 1950, 23 : 385-391. 